Pacific Journal of Mathematics

DUETS OF HYPERSIMPLE SETS 


\title{
SUBSETS OF HYPERSIMPLE SETS
}

\author{
R. G. DOWNEY
}

\begin{abstract}
Structural properties of r.e. sets often have degree theoretic consequences, particularly concerning degrees of supersets. It is our intention to show that such properties can have interesting ramifications on the degrees of subsets, by showing that no hypersimple r.e. set has the universal splitting property (USP). We also show that there are, however, simple sets (indeed, low and promptly simple sets) with USP and thus USP is not invariant under automorphisms of the lattice of r.e. sets.
\end{abstract}

1. Introduction. An r.e. sequence of disjoint nonempty canonical finite sets is called a strong array. Post [Po] defined an r.e. set $A$ to be hypersimple if it was coinfinite and given any infinite strong array $\left\{D_{x}\right\}_{x \in W}$, then for some $x, D_{x} \subset A$. Hypersimple sets were first constructed by Dekker [De] who showed that they exist in each r.e. degree, and have since been extensively studied.

A pair of r.e. sets $A_{1}, A_{2}$ is said to split an r.e. set $A$ (written $A_{1} \sqcup A_{2}=A$ ) if $A_{1} \cup A_{2}=A$ and $A_{1} \cap A_{2}=\varnothing$. Following Lerman and Remmel [LR1, 2] we shall say an r.e. set $A$ has the universal splitting property (USP) if given any r.e. set $B \leq_{T} A$ there is an r.e. splitting of $A=A_{1} \sqcup A_{2}$ with $A_{1} \equiv_{T} B$. Sets with and without USP have recently been analysed in several papers, for example [AS2, AF, Dol,2, DW, LR1,2]. Lerman and Remmel [LR2] showed that the degrees containing non-USP r.e. sets were dense in the r.e. degrees. In [Do1], the author improved this to show that in fact every nonzero r.e. degree contained a non-USP r.e. set. The main result of this paper is an extension of this:

THEOREM. (i) No hypersimple r.e. set has USP.

(ii) Indeed, if $A$ is hypersimple and $B$ is an r.e. set with $\varnothing<{ }_{T} B \leq{ }_{T} A$ then there exists an r.e. set $Q$ with $Q \leq{ }_{T} B$ and such that if $A_{1} \sqcup A_{2}=A$ is an r.e. splitting of $A$ then $A_{1} \equiv_{T} Q$.

(iii) In particular if $A_{1} \sqcup A_{2}=A$ is an r.e. splitting of a hypersimple set then $A_{1}$ is non-USP.

We feel that this result is quite interesting since it connects two apparently unrelated notions and means that properties of supersets of r.e. sets can affect degrees of subsets. 
As a companion result, we shall prove that no obvious lattice theoretic extension seems possible. To obtain the sharpest formulation, say $A$ has the SUSP (strong USP) if given any pair of r.e. sets with $B_{1} \oplus B_{2}$ $\equiv_{T} A$ there exist r.e. sets $A_{1}, A_{2}$ with $A=A_{1} \sqcup A_{2}$ and $A_{i} \equiv_{T} B_{i}$ for $i=1,2$. Recall from Maass [Ma], that $A$ is promptly simple if $\operatorname{card}(\omega-A)=\infty$ and there exists a recursive function $f$ such that $W_{e}$ infinite implies that for some $s, \exists x\left(x \in\left(W_{e, s+1}-W_{e, s}\right) \cap A_{f(x)}\right)$.

Our result here is:

\section{THEOREM. There exist low promptly simple SUSP r.e. sets.}

As a corollary, following some work of Maass, we see that (S)USP is not invariant under automorphisms of the lattice of r.e. sets.

The author wishes to thank Jeff Remmel for several helpful conversations regarding this material. He also wishes to thank the referee for finding several errors in an early draft of this paper.

2. Notation and conventions. Our notation and terminology is for the most part standard and follows Soare [So1, 2,3], subject to several conventions we now describe. We denote r.e. sets by upper case roman letters (e.g. $U, V, W, \ldots$ ) and $T$-functionals by upper case greek letters $(\Phi, \Gamma, \Delta, \ldots)$. We denote the use function of a computation by $u(-)$. This is the maximum element used in a computation (-). We suppose that if $\Phi(A ; x) \downarrow$ then $u(\Phi(A ; x))>x$.

If $\left\{\Phi_{s}\right\}$ and $\left\{A_{s}\right\}$ are approximations we shall suppose that if $\Phi_{s}\left(A_{s} ; x\right) \downarrow$ and $\Phi_{t}\left(A_{t} ; x\right) \downarrow$ then $u\left(\Phi_{s}\left(A_{s} ; x\right)\right) \leq u\left(\Phi_{t}\left(A_{t} ; x\right)\right)$. We remark that all sets and degrees etc. will be r.e. unless specifically stated otherwise. We let " $\sqcup$ " denote union for disjoint sets so that $A \sqcup B=C$ means $A \cup B=C$ and $A \cap B=\varnothing$. Also $\langle$,$\rangle will denote a standard$ pairing. For an (r.e.) set $A, A^{(e)}$ denotes $\{\langle e, x\rangle: x \in \omega\}$. We let $A[x]=$ $\{z: z \leq x \& z \in A\}$. Finally we adopt the convention that all computations etc. are bounded by $s$ at stage $s+1$.

3. Results. In [Do1], the author showed that every nonzero r.e. degree contained a non-USP r.e. set. At the time, we felt that the proof was slightly unusual since what it really showed was the following:

Let $A=f(\omega)$ be a 1-1 enumeration of an r.e. nonrecursive set. Define the "filled in" set $B$ associated with $A$ as follows:

Stage 0. Set $B_{0}=\varnothing$ and $b_{i, 0}=i$ for all $i \in \omega$. 
Stage $s+1$. Set $B_{s+1}=B_{s} \cup\left\{b_{i, s}: f(s) \leq i \leq f(s)+s\right\}, b_{i, s+1}=b_{i, s}$ for $i<f(s)$ and $b_{i, s+1}=b_{i+s+1, s}$ otherwise.

Set $B=\bigcup_{s} B_{s}$.

Then in [Do1] we proved:

THEOREM 3.1 ([Do1]). If $B$ is constructed as above, then $B$ is non-USP.

Our reason for feeling that this proof was slightly unusual was that construction of a nonsplitting witness - an r.e. set $C$ such that if $E$ and $F$ are r.e. sets with $E \sqcup F=B$ then $C \not \equiv_{T} E$-was virtually independent of our construction of $B$.

It thus seems reasonable to conjecture that perhaps the proof of [Do1] turned on a property of $B$ rather than the particular construction of $B$. Now it is easy to see that $B$ is hypersimple. As our first result we shall show that hypersimplicity is enough to ensure a set is non-USP. We use a modification of the techniques of [Do1]. We show:

THEOREM 3.2. (i) No hypersimple r.e. set has USP.

(ii) Indeed, if $A$ is hypersimple and $B$ is an r.e. set with $\varnothing<{ }_{T} B \leq{ }_{T} A$ then there exists an r.e. set $Q \leq_{T} B$ such that if $A_{1} \sqcup A_{2}=A$ is an r.e. splitting of $A$, then $A_{1} \not \equiv_{T} Q$.

(iii) In particular, if $A_{1} \sqcup A_{2}$ is an r.e. splitting of a hypersimple set, then $A_{1}$ is non-USP.

Proof. Let $A$ be a given hypersimple r.e. set and $B$ an r.e. set with $\varnothing<{ }_{T} B \leq{ }_{T} A$. Let $g(\omega)=B$ be a 1-1 enumeration of $B$. We shall build a nonsplitting witness $Q$ for $A$ with $Q \leq_{T} B$ by simple permitting as in [Do1]. Let $\left(V_{e}, W_{e}, \Gamma_{e}, \Phi_{e}\right)$ list all 4-tuples consisting of disjoint pairs of r.e. sets and pairs of functionals. Please note here that we are using disjoint pairs of r.e. sets $\left(V_{e}, W_{e}\right)$ and we shall thus know that at no stage of the construction can $V_{e, s} \cap W_{e, s} \neq \varnothing$. We must ensure $Q$ satisfies the requirements

$R_{e}$ : One of the following fails:

(i) $W_{e} \sqcup V_{e}=A$.

(ii) $\Phi_{e}\left(W_{e}\right)=Q$.

(iii) $\Gamma_{e}(Q)=W_{e}$.

We shall now briefly discuss the strategy for the satisfaction of a single $R_{e}$. We shall satisfy the $R_{e}$ by followers. Each follower $x$ is assigned a target region $T(x) . T(x)$ will be a (finite) subset of $\omega$. Define the " $Q$ 
controllable" length of agreement via

$$
\begin{aligned}
1(e, s)=\max \left\{x: \forall y<x\left(\Phi_{e, s}\left(W_{e, s} ; y\right)=\right.\right. & Q_{s}(y) \& \\
\forall z\left(z \leq u\left(\Phi_{e, s}\left(W_{e, s} ; y\right)\right)\right. & \rightarrow \Gamma_{e, s}\left(Q_{s} ; z\right)=W_{e, s}(z) \& \\
& \left.\left.\left.\left(W_{e, s} \sqcup V_{e, s}\right)(z)=A_{s}(z)\right)\right)\right\} .
\end{aligned}
$$

Associated with the $R_{e}$ will be a marker $M(e, s)$. Roughly speaking, the position of $M(e, s)$ will denote the part of $Q$ currently devoted to attempts to satisfy $R_{j}$ for $j \leq e$. Our argument will be finite injury, and so we shall suppose that beyond some stage $s_{0}$ we need only change $M(e, s)$ for the sake of attacks on $R_{e}$ (and the $R_{j}$ for $j<e$ have ceased activity). Also $M(e, s)$ is monotone in both variables.

Now we attack $R_{e}$ as follows. First we wait for a number $x$ to occur in $\omega^{(e)}$ such that

(i) $1(e, s)>x$, and

(ii) $x>\max \left\{u\left(\Gamma_{e, s}\left(Q_{x} ; z\right)\right): z \leq M(e, s)\right\}$.

At this stage, we appoint $x$ as a follower of $R_{e}$ and reset $M(k, s+1)$ to be $M(k, s+1)=s+1$ for $k \geq e$, We then set $T(x)=\{z: M(e, s)<z$ $\leq M(e, s+1)\}$ and cancel all lower priority followers. There are now two key observations.

Observation 1. For all stages $t \geq s$ if we do not add any number $\leq x$ to $Q_{t}-Q_{s}$ and if $1(e, t)>x$, we know $W_{e, t}[G]=W_{e, s}[G]$ where $G=$ $u\left(\Phi_{e, s}\left(W_{e, s} ; x\right)\right)$. This will follow since we have reset $M(k, s+1)$ to $s+1$ for all $k \geq e$, and our conventions regarding $u(-)$ ensure that all followers of $R_{j}$ for $j \geq e$ appointed after stage $s$ must exceed $s+1$, and all the $R_{j}$ for $j<e$ have ceased activity.

Observation 2. For all stages $t \geq s$ if no number $<x$ is added to $Q_{t}-Q_{s}$, and if $\Gamma_{e}(Q)=W_{e}$ then $W_{e, t}[M(e, s)]=W_{e, s}[M(e, s)]$. This follows by the same reasoning as Observation 1 since we specifically chose $x>\max \left\{u\left(\Gamma_{e, s}\left(Q_{s} ; z\right)\right): z \leq M(e, s)\right\}$. In particular, provided $Q_{s}[x-1]$ $=Q[x-1]$ we know $W_{e}[M(e, s)]=W_{e, s}[M(e, s)]$ (provided that it is truly the case that $\left.\Gamma_{e}(Q)=W_{e}\right)$.

Our eventual aim is to enumerate $x$ into $Q$. To do so immediately would be useless since although it might create a temporary disagreement between $\Phi_{e, s}\left(W_{e, s} ; x\right)$ and $Q_{e, s+1}(x)$, the fact that we have changed $Q_{e, t}[s]$ allows $W_{e, t}[G] \neq W_{e, s}[G]$ (where $G=u\left(\Phi_{e, s}\left(W_{e, s} ; x\right)\right)$ ) for some $t \geq s$.

However, it is important to note that if we added $x$ to $Q_{t}-Q_{s}$ but kept $Q_{t}[x-1]$ unchanged then Observation 2 tells us that although $W_{e, t}[G] \neq W_{e, s}[G]$ we do know that $W_{e, t}[M(e, s)]=W_{e, s}[M(e, s)]$. That 
is, $W_{e}$ may change, but only above $M(e, s)$. Thus as in [Do1], our idea is not to enumerate $x$ into $Q_{t}-Q_{s}$ until a stage where it is impossible for $W_{e, t}[G] \neq W_{e, s}[G]$ and yet $W_{e, t}[M(e, s)]=W_{e, s}[M(e, s)]$.

For our purposes it would surely suffice to find a stage $t>s$ such that

(i) $T(x) \subset A_{t}$, and

(ii) $1(e, t)>x$, and

(iii) $Q_{t}[x]=Q_{s}[x]$.

By Observation 1, if such a stage occurs, then if $W_{e}$ and $V_{e}$ truly split $A$ we know that $W_{e, t}[G]=W_{e, s}[G]$ and for all $z$ if $M(e, s)<z \leq G$, then $z \in W_{e}$ iff $x \in W_{e, s}$. (Any such $z$ not in $W_{e, s}$ must have entered $V_{e}$ ) In our construction, when such a stage $t$ occurs we shall declare $x$ as confirmed. We shall argue that once $x$ is confirmed if we ever get a chance to enumerate $x$ into $Q$ we shall ensure that

(a) either $\Phi_{e}\left(W_{e} ; x\right)=0 \neq 1=Q(x)$, or

(b) $\Gamma_{e}\left(Q_{e} ; z\right) \neq W_{e}(z)$ for some $z \leq M(e, s)$.

The remainder of the proof is an appeal to the hypersimplicity of $A$ to ensure that if $R_{e}$ fails to be met, infinitely many followers get confirmed. (The $\{T(x)\}$ form a strong array.) We then argue that if we do not get to win a confirmed follower, then $B$ is recursive contrary to hypothesis.

We shall now give the formal details of the argument although the reader-especially the one familiar with [Do1]-may prefer to supply these details for himself.

We shall say $R_{e}$ requires attention at stage $s+1$ if $e$ is least with one of the following options holding.

(3.3) $R_{e}$ has a follower $x$ which is confirmed and $g(s) \leq x$.

(3.4) Not (3.3), and there is a number $x \in \omega^{(e)}$ such that

(a) $1(e, s)>x$, and

(b) $x>\max \left\{u\left(\Gamma_{e, s}\left(Q_{s} ; z\right)\right) ; z \leq M(e, s)\right\}$.

\section{CONSTRUCTION}

Stage 0. All $T(x)$ for $x \in \omega$ are undefined. We set $M(e, 0)=0$ for all $e \in \omega$.

Stage $s+1$. Step 1. For each pair $x$ and $j$, if $x$ is a follower of $R_{j}$ and $x$ is not already confirmed, then if

(i) $T(x) \subset A_{s}$, and

(ii) $1(j, s)>x$, declare $x$ as confirmed.

Step 2. Find the least $e$ (if any) such that $R_{e}$ requires attention. Cancel all target regions and followers of $R_{j}$ for $j>e$. Adopt the appropriate case below. 
Case 1 (3.3) holds. Find the least such $x$. Set $Q_{s+1}=Q_{s} \cup\{x\}$. Cancel all followers $y$ of $R_{e}$ with $y \neq x$.

Case 2 (3.4) holds. Appoint $x$ as a follower of $R_{e}$. Set $M(k, s+1)=$ $s+1$ for $k \geq e$. Now set $T(x)=\{z: M(e, s)<z \leq M(e, s+1)\}$. Note that $T(x) \neq \varnothing$.

End of Construction.

Verification. Evidently $Q \leq_{T} B$ since numbers enter $Q_{s+1}-Q_{s}$ only when smaller ones enter $B_{s+1}-B_{s}$ : to decide if $x \in Q$ find the least stage $s$ where $\forall t>s(g(t)>x)$ then $x \in Q$ iff $x \in Q_{s+1}$.

As in [Do1] the argument is finite injury. Let $s_{0}$ be the least stage such that $\forall j<e \forall s>s_{0}\left(R_{j}\right.$ does not receive attention at stage $\left.s\right)$. Since $M(j, s) \neq M(j, s+1)$ only when some $R_{i}$ for $i \leq j$ receives attention, this means that $\forall j<e \forall s>s_{0}\left(M(j, s)=M\left(j, s_{0}\right)\right)$. Let $M=M\left(e, s_{0}\right)$ $=\max \left\{M\left(j, s_{0}\right): j \leq e\right\}$. By our cancellation procedure-when the $R_{j}$ receive attention-we may suppose $R_{e}$ has no follower at stage $s_{0}$.

LEMMA. If (3.3) holds for some follower $x$ of $R_{e}$ at some stage $s_{1}>s_{0}$ then

(i) $R_{e}$ is met at stage $s_{1}+1$,

(ii) $\forall t \geq s_{1}\left(R_{e}\right.$ does not require attention at stage $\left.t\right)$,

(iii) $\forall t>s_{1}\left(M(e, t)=M\left(e, s_{1}\right)\right)$.

Proof. Let $s_{1}$ be the least stage $>s_{0}$ where (3.3) pertains to any $x$ following $R_{e}$. Now $x$ was appointed at some stage $s_{2}+1$ with $s_{0}+1<$ $s_{2}+1<s_{1}$ (by (3.4)). By definition, we know that

(a) $1\left(e, s_{2}\right)>x$, and

(b) $x>\max \left\{u\left(\Gamma_{e, s_{2}}\left(Q_{s_{2}} ; z\right)\right): z \leq M\left(e, s_{2}\right)\right\}$.

When $x$ was appointed, we reset $M\left(k, s_{2}+1\right)=s_{2}+1$ for all $k \geq e$, and we cancelled all followers of $R_{j}$ for $j>e$. By choice of $s_{0}$ and the fact that the $M(k, s)$ are clearly monotone, it follows that

$$
Q_{s_{1}}\left[s_{2}+1\right]=Q_{s_{2}}\left[s_{2}+1\right] \text {. }
$$

The important point here is that after stage $s_{2}+1$ followers that are appointed must exceed $s_{2}+1$. Also since $s_{1}$ is the least stage at which (3.3) pertains we also know that no numbers $\leq s_{2}+1$ have entered $Q_{s_{1}}-Q_{s_{2}}$ for the sake of $R_{e}$.

Now since (3.3) pertains to $x$ it follows that $x$ must be confirmed, and so there is a stage $t$ with $s_{2}+1<t \leq s_{1}$ with

(i) $1(e, t)>x$, and

(ii) $T(x) \subset A_{t}$. 
Now by (3.5) and the facts that $1\left(e, s_{2}\right)>x$ and $1(e, t)>x$, we see

$$
W_{e, t}\left[u\left(\Phi_{e, s_{2}}\left(W_{e, s_{2}} ; x\right)\right)\right]=W_{e, s_{2}}\left[u\left(\Phi_{e, s_{2}}\left(W_{e, s_{2}} ; x\right)\right)\right] \text {. }
$$

However, we specifically chose $T(x)$ as

$$
T(x)=\left\{z: M\left(e, s_{2}\right)<z \leq s_{2}+1\right\} .
$$

Thus as $W_{e}$ and $V_{e}$ are disjoint and $T(x) \subset A$, it must be that

(3.7) For all $z \in W_{e}$ if $z \leq u\left(\Phi_{e, t}\left(W_{e, t} ; x\right)\right)$ and $z \notin W_{e, t}$ then $z \leq M\left(e, s_{2}\right)$.

The points to note here are that since $1(e, t)>x$ we must have that

$$
A_{t}[u]=W_{e, t}[u] \sqcup V_{e, t}[u] \quad \text { where } u=u\left(\Phi_{e, t}\left(W_{e, t} ; x\right)\right),
$$

and furthermore $T(x) \subset A_{t}$.

Now by choice of $x$ in (3.4) we know that

$$
x>u\left(\Gamma_{e, s_{2}}\left(Q_{s_{2}} ; y\right)\right) \text { for all } y \leq M\left(e, s_{2}\right) .
$$

By (3.5) we know that this means that

$$
x>u\left(\Gamma_{e, s_{1}}\left(Q_{s_{1}} ; y\right)\right) \text { for all } y \leq M\left(e, s_{2}\right) .
$$

When (3.3) pertains and so $R_{e}$ receives attention via $x$ at stage $s_{1}+1$ we not only add $x$ to $Q$ but also cancel all other followers of $R_{e}$. By choice of $s_{0}$ and $s_{1}$ this means that $Q_{s_{1}}[x-1]=Q[x-1]$. By (3.5) and (3.9) this ensures that

(3.10) For all $y \leq M\left(e, s_{2}\right), \Gamma_{e, s_{2}}\left(Q_{s_{2}} ; y\right)=\Gamma_{e, s_{1}}\left(Q_{s_{1}} ; y\right)=\Gamma_{e}(Q ; y)$.

Now (3.3) pertains to $x$ at stage $s_{1}$. There are two cases.

Case 1. $\exists q\left(t \leq q \leq s_{1} \& 1(e, q) \leq x\right)$. In this case the only possibility is that some number $z \leq M\left(e, s_{2}\right)$ has entered $W_{e, s_{1}}-W_{e, t}$. (Again we use the disjointness of $W_{e}$ and $V_{e}$ here.) But now (3.10) ensures that

$$
\Gamma_{e}(Q ; z)=0 \neq W_{e}(z) \text {. }
$$

Case 2. $\forall q\left(t \leq q \leq s_{1} \rightarrow 1(e, q)>x\right)$. By (3.6) and (3.7) we know that

$$
0=W_{e, s_{1}}\left[u\left(\Phi_{e, s_{1}}\left(W_{e, s_{1}} ; x\right)\right)\right]=W_{e, s_{2}}\left[u\left(\Phi_{e, s_{2}}\left(W_{e, s_{2}} ; x\right)\right)\right]
$$

hence we create a disagreement

$$
\Phi_{e, s_{1}}\left(W_{e, s_{1}} ; x\right)=0 \neq 1=Q_{s_{1}+1}(x) .
$$


This disagreement will remain unless

$$
W_{e, s_{1}}\left[u\left(\Phi_{e, s_{2}}\left(W_{e, s_{2}} ; x\right)\right)\right] \neq W_{e, s_{3}}\left[u\left(\Phi_{e, s_{2}}\left(W_{e, s_{2}} ; x\right)\right)\right]
$$

for some (least) $s_{3}>s_{1}$. But if this occurs, by (3.7) we know $W_{e, s_{3}}\left[M\left(e, s_{2}\right)\right] \neq W_{e, s_{1}}\left[M\left(e, s_{2}\right)\right]$ and hence for some $z \leq M\left(e, s_{2}\right)$ we know

$$
\Gamma_{e}(Q ; z) \neq W_{e}(z) .
$$

It follows that $R_{e}$ is met. Also since $1(e, s)<M\left(e, s_{2}+1\right)$ for all $s>s_{1}+1$ it follows that $R_{e}$ will never again receive attention. This ensures that $M\left(e, s_{1}\right)=M(e, s)$ for all $s>s_{1}$ as well. Our result now follows.

Conclusion of the proof of (3.2). Thus it suffices to show that (3.3) pertains. Suppose (3.3) does not pertain after stage $s_{0}$, and $R_{e}$ receives attention infinitely often or fails to be met. Then as $1(e, s) \rightarrow \infty$ (3.4) must pertain at some stage $s_{1}>s_{0}$. At this stage we appoint a pair $\left(T\left(x_{1}\right), x_{1}\right)$ consisting of a follower and its target region. In like fashion, (3.4) must again pertain to eventually get an infinite increasing list

$$
\left(T\left(x_{1}\right), x_{1}\right), \quad\left(T\left(x_{2}\right), x_{2}\right), \ldots
$$

with $x_{1}<x_{2}<\cdots$.

Evidently $\left\{T\left(x_{i}\right)\right\}_{i \in \omega}$ is a strong array. Hence as $A$ is hypersimple for infinitely many $x_{i}$ we have $T\left(x_{i}\right) \subset A$. As $1(e, s) \rightarrow \infty$, each such $x_{i}$ must eventually get confirmed. We show now that $g(\omega)=B$ is a recursive set, contrary to hypothesis. Let $z \in \omega$. Let $s=s(z)$ be the least stage with $s>s_{0}$ and such that there is a confirmed follower $x>z$ of $R_{e}$ at stage $s$. Then $z \in B$ iff $z \in B_{s}$ since (3.3) does not pertain. The result now follows.

It therefore follows that possessing a fairly sparse lattice of supersets (e.g. a maximal set) imposes strong degree-theoretic restrictions on the possible degrees of subsets. Further results along these lines were recently obtained by Mike Stob and the author in [DS2]. These results concerned splittings of maximal sets. In [DS2] it is shown - for example-that if $\mathbf{a}$ is any nonzero r.e. degree there exist r.e. degrees $\mathbf{b}$ and $\mathbf{c}$ with $\mathbf{0}<\mathbf{b}<\mathbf{a}$ and $\mathbf{0}<\mathbf{c}<$ a such that

(i) there exist r.e. disjoint sets $C$ and $D$ with $\operatorname{deg}(C)=\mathrm{c}$ and $C \sqcup D$ maximal, and

(ii) there do not exist disjoint r.e. sets $B$ and $E$ with $\operatorname{deg}(B)=\mathbf{b}$ and $B \sqcup E$ maximal (or $r$-maximal or $h h$-simple). 
We remark that (ii) and several extensions are established by techniques along the lines of those of (3.2). We also remark that the degrees of halves of splittings of maximal sets are particularly interesting, since in [DS2] it is also shown that the r.e. nonrecursive sets $A$ such that there is an r.e. $B$ with $B \not \equiv \varnothing$ with $A \cap B=\varnothing$ and $A \cup B$ maximal, form an orbit in the automorphism group of the lattice of r.e. sets. This is the first known elementarily definable orbit (containing members in all high degrees) satisfying degree theoretic properties like those in (i) and (ii) above.

Before we continue we would like to give one (final!) construction of a non-USP set that we feel provides an interesting counterpoint to (3.2) and the [Do1] construction, both of which seem to work because large "blocks" of elements are put into $A$. Let $B$ be any nonrecursive r.e. set. Define $A$ as follows:

Stage 0. Let $f(\omega)=B$ be a 1-1 enumeration of $B$. Let $A_{0}=\varnothing$ and $b_{i, 0}=i$ for all $i \in \omega$.

$$
\begin{aligned}
& \text { Stage } s+1 \text {. Set } A_{s+1}=A_{s} \cup\left\{b_{f(s), s}\right\} \\
& \text { and set } \\
& \qquad b_{i, s+1}= \begin{cases}b_{i, s} & \text { for } i<f(s) \\
b_{i+f(s)+s+1}, & \text { otherwise. }\end{cases}
\end{aligned}
$$

Here the reader should think of the $b_{i, s}$ as markers placed on some, but not all, members of $\overline{A_{s}}$. Now define $A=\bigcup_{s} A_{s}$. Then via exactly the same argument as [Do1], $A$ is non-USP.

It is interesting to speculate on other properties of $A$ constructed as above. Ambos-Spies [AS2] has shown that for a particular choice of $B$ it is possible to make $A$ complete but to also have the property that if $A_{1} \sqcup A_{2}=A$ is an r.e. splitting of $A$ then one of $A_{1}$ or $A_{2}$ is low. In particular the author would like to know if it is possible to choose $B$ so that $A$ is speedable (cf. [So3]) but have the Ambos-Spies property that if $A_{1} \sqcup A_{2}=A$ is an r.e. splitting of $A$ then one of $A_{1}$ or $A_{2}$ is low. (It is not known if all speedable r.e. sets can be split into a pair of speedable subsets. This is an apparently difficult open question due to Jeff Remmel.)

Returning now to the possible lattice properties of non-USP r.e. sets, we might be tempted to conjecture that perhaps simple sets might be non-USP or alternatively suggest that USP might be invariant under automorphisms of the lattice of r.e. sets. To dispose of these possibilities, we prove Theorem 3.11 below.

THEOREM 3.11. There exist low promptly simple SUSP r.e. sets. 
Proof. The proof of this result is rather more difficult than (3.2) and it relies on a tree of strategies argument. We shall build $A=\cup_{s} A_{s}$ in stages to satisfy $\operatorname{card}(\omega-A)=\infty$ and

$$
\begin{aligned}
& P_{e}: \operatorname{card}\left(W_{e}\right)=\infty \text { implies } \exists y, s\left(y \in W_{e, s+1}-W_{e, s} \& y \in A_{s}\right) \\
& H_{e}: \exists^{\infty}\left(\Phi_{e, s}\left(A_{s} ; e\right) \downarrow\right) \text { implies } \Phi_{e}(A ; e) \downarrow \\
& N_{e}: \text { If } \Phi_{e}(A)=W_{e} \oplus V_{e} \text { and } \Gamma_{e}\left(W_{e} \oplus V_{e}\right)=A \\
& \quad \text { then for some r.e. sets } Q_{e}, R_{e} \text { we have } \\
& \quad Q_{e} \sqcup R_{e}=A, Q_{e} \equiv{ }_{T} W_{e} \text { and } R_{e} \equiv_{T} V_{e} .
\end{aligned}
$$

(Here we work over 4-tuples $\left(\Phi_{e}, \Gamma_{e}, W_{e}, V_{e}\right)$ as in (3.2).) Thus $P_{e}$ ensures that $A$ is promptly simple via the identity function, and $H_{e}$ is the standard lowness requirement as in [So2]. Thus for the $H_{e}$ we define a restraint

$$
r(e, s)= \begin{cases}1+u\left(\Phi_{e, s}\left(A_{s} ; e\right)\right) & \text { if } \Phi_{e, s}\left(A_{s} ; e\right) \downarrow \\ 0 & \text { otherwise }\end{cases}
$$

and let $R(e, s)=\max \{r(j, s): j \leq e\}$. Now, given that we argue the positive restraints are finitary, and if we only add $y$ to $A_{s+1}-A_{s}$ for the sake of $P_{j}$ for $j \geq e$ if $y \geq R(e, s)$, the standard argument will show $A$ is low as in Soare [So2]. Hence we will not discuss these further, but refer the reader to [So2] if he wishes further amplification. We also will not consciously make $\operatorname{card}(\omega-A)=\infty$ but simply remark that this is a simple enough matter to arrange (and will actually come out of the construction).

The crucial dynamics of the construction involve the interaction of the $N_{e}$ requirements and the satisfaction of the $P_{e}$. We first briefly attempt to describe our method of satisfaction of the $N_{e}$, at least for a single $N_{e}$.

Define the " $A$ controllable" length of agreement as

$$
\begin{aligned}
1(e, s)=\max \{x: \forall y<x & {\left[\Gamma_{e, s}\left(W_{e, s} \oplus V_{e, s} ; y\right)=A_{s}(y)\right.} \\
& \& \forall z\left(z \leq u\left(\Gamma_{e, s}\left(W_{e, s} \oplus V_{e, s} ; y\right)\right)\right. \\
& \left.\left.\left.\rightarrow \Phi_{e, s}\left(A_{s} ; z\right)=\left(W_{e, s} \oplus V_{e, s}\right)(z)\right)\right]\right\} .
\end{aligned}
$$

Our strategy was arrived at by a series of approximations, and it would perhaps be useful to use this approach to describe our strategy. The crudest attempt to satisfy the $N_{e}$ is to define a recursive series of markers $x(e, 0), x(e, 1), \ldots$ and for $x=x(e, i)$ to simply wait until the first stage $s$ occurs where $1(e, s)>x$. We then set $s(x)=s$ and try to ensure that

$$
\begin{aligned}
& \forall t>s(1(e, t)>1(e, s) \text { implies } \\
& \left.\quad\left(W_{e, t}[s(x)]=W_{e}[s(x)] \text { iff } Q_{e, t}[x]=Q_{e}[x]\right)\right) .
\end{aligned}
$$


Of course we must treat $R_{e}$ and $V_{e}$ similarly. Assuming that $x(e, i)$ $\rightarrow \infty$, (3.12) will clearly ensure that $W_{e} \equiv{ }_{T} Q_{e}$.

We attempt to implement this strategy by monitoring the progress of $W_{e, t}[s(x)]$ and $V_{e, t}[s(x)]$ at e-expansionary stages; that is, stages where $1(e, s)>m 1(e, s)$ where $m 1(e, s)=\max \{1(e, t): t<s\}$. The idea is then that if $W_{e, t}[s(x)] \neq W_{e, q}[s(x)]$-where $q=\max \left\{t^{\prime}<t \& 1\left(e, t^{\prime}\right)>\right.$ $\left.m 1\left(e, t^{\prime}\right)\right\}$-hopefully we have arranged matters so that $A_{t}[x] \neq A_{q}[x]$. Then we can ensure $Q_{e, t}[x] \neq Q_{e, q}[x]$ by enumerating into $Q_{e}$ some element $y \leq x$ with $y \in A_{t}-A_{q}$.

The reader should note that even in this crude approximation, the fact that $A_{t}[x] \neq A_{q}[x]$ is really not enough. Perhaps $y \leq x$ is the unique element to enter $A_{t}-A_{q}$ and $y=x(e, j)$ for some $x(e, j)<x$. Now since (3.12) is an "iff" condition for it to be legal for us to add $y$ into $Q_{e}$, it must also be the case that $W_{e, t}[s(y)] \neq W_{e, q}[s(y)]$.

It is also important to note here that the dynamics of the construction force us to proceed by first adding elements to $A$, then waiting until the next $e$-expansionary stage to decide which sets $Q_{e}$ or $R_{e}$ to put these new elements into. (In modern terminology this is essentially "closing a gap".) The reason for this order is that we must wait to see which of $W_{e}$ or $V_{e}$ changes to decide which set to place the appropriate elements into. (It might seem feasible to alternatively wait till the end of a gap and see which of $W_{e}$ or $V_{e}$ changes, and then perhaps add to $A$ some further elements as traces to the $V_{e}$ and $W_{e}$ change. This strategy seems to fail since (for example) $R(e, s)$ might change in intervening stages.)

Now even at the local level, if our strategy has any hope of working, we must try to ensure that whenever $W_{e}[s(x)]$ has a chance of changing, we need to also ensure that $A[x]$ has a chance of changing also. This is primarily achieved by two devices. First we use a sort of "waiting and cancellation" procedure in some ways along the lines of a "contiguous degree" construction (cf. Stob [St]). As in the confirmation procedure of (3.2), this waiting and cancellation procedure attempts to clear $A$ of potentially injurious numbers $z$ with $z<u\left(\Phi_{e, s}\left(A_{s} ; g\right)\right)$ for all $g \leq s(x)$ and thus tries to force $W_{e}[s(x)]$ to be able to change only if $Q_{e}[x]$ changes. This type of device has been used in several other settings and its origins may be traced back to Ladner [Ld2]. (In our construction this is the reason for the $b(\sigma, s)$ function.) The second device we use is to replace the single $x(e, i)$ marker by a finite block $\{x(e, i, 1), \ldots, x(e, i, n)\}$ of markers. Roughly speaking this is forced upon us because of the interactions of $W_{e}$ or $V_{e}$ changes. The point is that the waiting-cancellation procedure ensures us to some $W_{e}$ or $V_{e}$ change, but if both sets change, perhaps $W_{e}$ changes through $s(x)$ and $V_{e}$ changes through $s(y)$, but $y$ is 
very much smaller than $x$. This will be good from $R_{e}$ 's point of view (because it will be that $A[y]$ changes) but is bad from $Q_{e}$ 's point of view since we cannot use $y$ (or $y+1$, etc.) to add to $Q_{e}$ if $W_{e}$ does not change through $s(y)$. The use of a block of markers essentially ensures that there will be some appropriate element in $A_{t}-A_{q}$ - "one from block $x$ " - that we shall be able to add to $Q_{e}$. It is essentially possible to preset this block in advance because we will know precisely the maximum number of "injuries" any block can suffer in the same way as Post's simple set construction.

The final problems we must overcome are due to our lack of knowledge of which $1(e, s) \rightarrow \infty$ and thus how to combine strategies for the various $N_{e}$. This problem is much the same as, say, a minimal pair construction. We choose to overcome this problem using a tree of strategies argument. We use $T=2^{<\omega}$ to encode certain "guesses" as to the behaviour of the various $N_{j}$ for $j \leq e$ and $e \in \omega$. Hence in particular in the place of $Q_{e}$ and $R_{e}$ and $x(e, i, n)$ we shall use certain "guessed" versions which we denote by $Q_{\sigma}, R_{\sigma}$ and $x(\sigma, i, n(\sigma))$ where $\sigma$ has length $e+1$. It suffices to argue for some $\sigma$ the " $\sigma$-strategy" is successful. For more on tree arguments we refer the reader to [So3] and [So4, Chapter XIV]. The conscious use of trees was introduced by Lachlan [La3].

We now give the formal details of the argument. We refer to $\sigma \in 2^{<\omega}$ as guesses. Let $\operatorname{lh}(\sigma)$ denote the length of $\sigma$. Let $\sigma \subset \tau$ denote $\sigma$ is an initial segment of $\tau$. We define the usual lexicographic ordering $\leq_{L}$ via $\sigma \leq_{L} \tau$ iff $\sigma \subset \tau$ or $\exists \gamma\left(\gamma^{\wedge} 0 \subset \sigma \& \gamma^{\wedge} 1 \subset \tau\right)$. Here we are denoting the concatenation of $\sigma$ and $\tau$ by $\sigma^{\wedge} \tau$.

As we remarked earlier, for the waiting-cancellation strategy, it will be convenient in the construction to make use of a marker $b(\sigma, s)$. We refer to this as the $\sigma$-boundary. Roughly speaking, the $\sigma$-boundary (for $\ln (\sigma)=$ $e+1$ ) indicates the amount of $A$ that $N_{e}$ believes is "covered" as far as guess $\sigma$ is concerned. Finally in the construction we shall use the word "large fresh number". This is taken to mean a number to exceed all numbers previously considered in the construction; and saves on notation.

We define a $\sigma$-stage by induction on $\operatorname{lh}(\sigma)$ :

(i) Every stage $s$ is an $\varnothing$-stage.

(ii) If $s$ is a $\tau$-stage and $\operatorname{lh}(\tau)=e$ then if $1(e, s)>\max \{1(e, t): t$ is a $\tau$-stage and $t<s\}$ and $1(e, s)>b\left(\tau^{\wedge} 0, s\right)$ then $s$ is a $\tau^{\wedge} 0$-stage.

Otherwise $s$ is a $\tau^{\wedge} 1$-stage.

Let $\sigma_{s}$ denote the unique path of length $s$ such that $s$ is a $\sigma_{s}$-stage. We shall say $P_{e}$ requires attention at stage $s+1$ if one of the following options holds. 
$P_{e}$ has a follower $x>R(e, s)$ such that $\exists y\left(y \in W_{e, s+1}-W_{e, s} \& y>x\right)$.

$P_{e}$ has no follower $x$ with $x>R(e, s)$ such that $x$ has guess $\sigma_{s}$, and furthermore $P_{e}$ has not been declared satisfied.

The reader-especially the one already familiar with tree arguments - should note that (3.13) means that $P_{e}$ can receive attention via a follower $x$ with guess $\sigma$ at other than $\sigma$-stages. This seems crucial if we are needing to achieve prompt simplicity rather than just simplicity.

\section{CONSTRUCTION.}

Stage 0. Set $A_{0}=Q_{\sigma, 0}=R_{\sigma, 0}=\varnothing$ for all $\sigma \in 2^{<\omega}$. For all such $\sigma$, set $b(\sigma, 0)=0$ and declare $x(\sigma, i, j)$ and $s(\sigma, i)$ as undefined for all $i$ and $j$.

Stage $s+1$.

Step 1 (Cancellation). Cancel all followers with guess $\tau$, all $x(\tau, i, j)$ and all $s(\tau, i)$ for all $\tau \nless{ }_{L} \sigma_{s}$. For all such $\tau$ set $R_{\tau, s+1}=\varnothing$ and $Q_{\tau, s+1}=\varnothing$.

Step 2 (Trace assignment). For each $\tau^{\wedge} 0 \subset \sigma_{s}$, find the least $i$ (if any) with $x\left(\tau^{\wedge} 0, i\right)$ defined but $s\left(\tau^{\wedge} 0, i\right)$ not yet defined. Define $s\left(\tau^{\wedge} 0, i\right)=z$ where $z=\max \left\{u\left(\Gamma_{e, s}\left(W_{e, s} \oplus V_{e, s} ; p\right)\right): p \leq 1(e, s)\right\}$ for $e=1 \mathrm{~h}(\tau)$.

Step 3 (Marker appointment). For each $\tau^{\wedge} 0 \subset \sigma_{s}$ define a new block of markers (in order of $\tau^{\wedge} 0$ ) as follows.

Find the least $i$ with block $x\left(\tau^{\wedge} 0, i\right)$ currently undefined. Find $n=3 s+4$ large fresh numbers $y_{1}, \ldots, y_{n}$ and appoint the current marker block as

$$
x\left(\tau^{\wedge} 0, i\right)=\left\{x\left(\tau^{\wedge} 0, i, 1\right), \ldots, x\left(\tau^{\wedge} 0, i, n\right)\right\}
$$

with $x\left(\tau^{\wedge} 0, i, j\right)=y_{j}$. (The number $3 s+4$ is not critical here, it is simply one we know will be large enough to ensure the block is not used up.) We remark that we may in the next step add two further numbers (followers of some $\left.P_{j}\right)$ to each such $x\left(\tau^{\wedge} 0, i\right)$ we have defined in this step. This will be done for technical convenience only.

REMARK. We remark that we should possibly use a notation $x\left(\tau^{\wedge} 0, i\right)(s)$ to indicate $x\left(\tau^{\wedge} 0, i\right)$ at stage $s$, but we feel that this will be clear from context, since once $x\left(\tau^{\wedge} 0, i\right)$ is defined it remains fixed or else becomes undefined later. Furthermore, after some finite stage we will soon see that $x\left(\tau^{\wedge} 0, i\right)$ remains fixed for “correct" $\tau^{\wedge} 0$. 
Step 4. Find the least $e$ such that $P_{e}$ requires attention. Adopt the appropriate case below.

Case 1 (Follower appointment). (3.14) holds; find a large fresh number $y$ and appoint $y$ as a follower of $P_{e}$ with guess $\sigma$ where $\sigma \subset \sigma_{s}$ and $\operatorname{lh}(\sigma)=e+1$. Cancel all followers with guesses $\gamma \supset \sigma$ and $\gamma \neq \sigma$. Now for each $\tau^{\wedge} 0 \subset \sigma_{s}$ find the set $x\left(\tau^{\wedge} 0, i\right)$ defined in Step 4, and enumerate $y$ and $y+1$ into $x\left(\tau^{\wedge} 0, i\right)$ (so that now $x\left(\tau^{\wedge} 0, i\right)$ has $3 s+6$ elements). Go to Step 5.

Case 2 (Satisfaction). (3.13) holds. Enumerate $x, x+1$ and $y$ into $A_{s+1}-A_{s}$. Let $x_{1}, \ldots, x_{m}$ list those marker blocks currently defined and satisfying $\forall z \in x_{i}(z>x+1)$. (Remark: this will consist of those blocks currently defined and appointed after the stage $x$ was appointed.) For each such block find the least two elements $g_{i}, h_{i}$ with

$$
g_{i}, h_{i} \in x_{i}-A_{s} \quad \text { (These will not be followers.) }
$$

Also enumerate $\left\{g_{i}, h_{i}: 1 \leq i \leq m\right\}$ into $A_{s+1}-A_{s}$. Cancel all (remaining) followers of $P_{j}$ for $j>e$. Declare $P_{e}$ as satisfied (forever).

REMARK. The reader should note that when some follower $x$ of $P_{e}$ is enumerated into $A$ we perhaps cancel some follower $z>x$. Now perhaps $z \in x\left(\gamma^{\wedge} 0, j\right)$ for several $j$. It really will not matter whether or not we regard $z$ as still a member of $x\left(\gamma^{\wedge} 0, j\right)$ after it is cancelled. The crucial fact will be that $z$ is no longer a follower once it is cancelled. For technical reasons if $x\left(\gamma^{\wedge} 0, j\right)$ is defined it is easiest to regard $z$ as still a member of $x\left(\gamma^{\wedge} 0, j\right)$ but ask that $z$ never be added to $A$ unless $z$ is added to $A$ for the sake of some $P_{k}$ for $k<e$. A counting argument ensures that this is possible.

Step 5 (Defining boundaries). Find a large fresh number $z$ and define $b\left(\tau^{\wedge} 0, s\right)=z$ for each $\tau^{\wedge} 0 \subset \sigma_{s}$.

Step 6 (Recovery). In this step we attempt to build our splitting of $A$. Thus for each $\sigma=\tau^{\wedge} 0 \subset \sigma_{s}$ perform the following recovery step: let $1 \mathrm{~s}(\sigma, s)$ denote last $\sigma$-stage $<s$, namely

$$
\operatorname{ls}(\sigma, s)=\max \{0, t: t \text { is a } \sigma \text {-stage and } t<s\} .
$$

Let $t=\operatorname{ls}(\sigma, s)$ and let $e=\operatorname{lh}(\tau)$. Now if $x\left(\tau^{\wedge} 0, i\right)$ is currently defined let $m x\left(\tau^{\wedge} 0, i\right)$ denote the largest member of $x\left(\tau^{\wedge} 0, i\right)$. Now find the least $i$ and $j$ (if any) such that $s\left(\tau^{\wedge} 0, i\right)$ and $s\left(\tau^{\wedge} 0, j\right)$ were defined at 
stage $t$ and

$$
\begin{array}{ll}
\text { (i) } & A_{s}\left[m x\left(\tau^{\wedge} 0, i\right)\right] \neq A_{t}\left[m x\left(\tau^{\wedge} 0, i\right)\right] \text { and } \\
& W_{e, s}\left[s\left(\tau^{\wedge} 0, i\right)\right] \neq W_{e, t}\left[s\left(\tau^{\wedge} 0, i\right)\right], \text { and } \\
\text { (ii) } & A_{s}\left[m x\left(\tau^{\wedge} 0, j\right)\right] \neq A_{t}\left[m x\left(\tau^{\wedge} 0, j\right)\right] \text { and } \\
& V_{e, s}\left[s\left(\tau^{\wedge} 0, j\right)\right] \neq V_{e, t}\left[s\left(\tau^{\wedge} 0, j\right)\right],
\end{array}
$$

There are several cases to consider:

Case 1. Neither $i$ nor $j$ exists. In this case set $Q_{\sigma, s+1}=Q_{\sigma, s} \cup\left(A_{s}-\right.$ $\left.\left(Q_{\sigma, s} \cup R_{\sigma, s}\right)\right)$ and $R_{\sigma, s+1}=R_{\sigma, s}$.

Case 2. $i$ exists but $j$ does not. As in Case 1 .

Case 3. $j$ exists but $i$ does not. In this case set $R_{\sigma, s+1}=R_{\sigma, s} \cup\left(A_{s}\right.$ - $\left.\left(Q_{\sigma, s} \cup R_{\sigma, s}\right)\right)$ and $Q_{\sigma, s+1}=Q_{\sigma, s}$. For the remaining cases we have that $i$ and $j$ both exist.

Case 4. $i=j$. In this case let $y_{1}$ and $y_{2}$ be the least two numbers to enter $A_{s}-A_{t}$.

We claim two such numbers exist, and in fact $y_{2}=y_{1}+1$ and $y_{1}$ is a follower, this being checked in the verification. We similarly claim that not only are both $y_{1}$ and $y_{2}<m x\left(\tau^{\wedge} 0, i\right)$, but in fact $y_{1}$ and $y_{2}$ are members of $x\left(\tau^{\wedge} 0, i\right)$.

Assuming this claim, we shall set $R_{\sigma, s+1}=R_{\sigma, s} \cup\left\{y_{1}\right\}$ and $Q_{\sigma, s+1}=$ $Q_{\sigma, s} \cup\left(A_{s}-\left(Q_{\sigma, s} \cup R_{\sigma, s+1}\right)\right)$.

Case 5. $i<j$. In this case we claim that

some number in block $x\left(\tau^{\wedge} 0, j\right)$ entered $A_{s}-A_{t}$.

Let $z$ be the least such number. We set $R_{\sigma, s+1}=R_{\sigma, s} \cup\{z\}$ and $Q_{\sigma, s+1}=Q_{\sigma, s} \cup\left(A_{s}-\left(Q_{\sigma, s} \cup R_{\sigma, s+1}\right)\right)$.

Case 6. $j<i$. As in Case 5 with the roles of $Q$ and $R$ reversed, noting that some $z$ in block $x\left(\tau^{\wedge} 0, i\right)$ entered $A_{s}-A_{t}$.

END OF CONSTRUCTION.

Verification. We first verify the $P_{e}$ and $H_{e}$ as these are fairly standard arguments.

Let $\beta$ denote the leftmost path. That is, $\varnothing \subset \beta$ and $\gamma \subset \beta$ implies $\gamma^{\wedge} 0 \subset \beta$ if $\exists^{\infty} s$ (s is a $\gamma^{\wedge} 0$-stage) otherwise $\gamma^{\wedge} 1 \subset \beta$. Now by construction (3.13) pertains to $P_{e}$ at most once. Hence $H_{e}$ will be met. By [So2] we now argue that $P_{e}$ is met. Let $\sigma \subset \beta$ with $\operatorname{lh}(\sigma)=e+1$ and let $s_{0}$ be a stage good for $\sigma$, namely

(i) $\forall s>s_{0}\left(\sigma \leq{ }_{L} \sigma_{s}\right)$,

(ii) $\forall j<e \forall s>s_{0}$ ( $s$ is a $\sigma$-stage implies $P_{j}$ does not receive attention at stage $s$ ), 
(iii) $\forall j<e \forall s>s_{0}\left((3.13)\right.$ does not pertain to $P_{j}$ at stage $\left.s\right)$,

(iv) $\forall s>s_{0} \forall j \leq e(r(j, s)=r(j))$.

Without loss, we might as well suppose $P_{e}$ has no followers. Now the next follower $x$ to be appointed to $P_{e}$ is clearly uncancellable. Thus at the least stage $s+1>s_{0}$ when some $y>x$ occurs in $W_{e, s+1}-W_{e, s}$, we will meet $P_{e}$.

The remainder of the verification is devoted to checking that our apparatus manages to meet the $N_{e}$. Thus again let $\sigma=\tau^{\wedge} 0 \subset \beta$ with $\operatorname{lh}(\sigma)=e+1$ and let $s_{0}$ be good for $\sigma$ as above. First observe that by our cancellation procedure we can suppose that $x(\sigma, 0)$ is undefined at stage $s_{0}$. Now by choice of $s_{0}$ and $\sigma$ it is clear that $\forall i \forall t>s_{0} \forall s>t(x(\sigma, i)$ defined at stage $t$ implies $x(\sigma, i)$ defined and unchanged at stage $s)$. Furthermore the same holds for $s(\sigma, i)$. Moreover as $1(e, s) \rightarrow \infty$ we see that for all $i \in \omega, x(\sigma, i)$ and $s(\sigma, i)$ eventually become (permanently) defined.

Suppose $x(\sigma, i)$ is defined at stage $t>s_{0}$. Then $x(\sigma, i)$ is now fixed and has at least $3 t+4$ elements. These elements can only be added to $A$ because of the action of a $P_{j}$ requirement, and by construction we only add these elements to $A$ ( $\leq 3$ at a time) when some follower $\leq m x(\sigma, i)$ enters $A$. By the way we appoint followers, all followers appointed after stage $t$ must exceed $m x(\sigma, i)$. A counting argument ensures that $x(\sigma, i) \not \subset$ $A$ (and so there are always markers in $x(\sigma, i)$ not yet in $A_{s}$ at any $s>s_{0}$ ). The remainder of the proof is given by a sequence of lemmas.

(3.15) LEMMA. Let $q$ and $t$ be o-stages with $q>t>s_{0}$ and such that $s(\sigma, i)$ is defined at stage $t+1$. Then $A_{t}[m x(\sigma, i)]=A_{q}[m x(\sigma, i)]$ implies $\left(W_{e, t} \oplus V_{e, t}\right)[s(\sigma, i)]=\left(W_{e, q} \oplus V_{e, q}\right)[s(\sigma, i)]$.

REMARK. Before we begin the formal proof of this lemma, the reader should note that the "guiding principle" in the verification of (3.15) and later lemmata is that although there are lots of numbers (such as markers) which can apparently injure computations due to their addition to $A$, they are only added to $A$ in response to-and at the same stage as-(smaller) followers being added to $A$. Markers never initiate such "injury"; only followers may do so.

Proof (of 3.15). Let $t$ and $q$ satisfy the hypotheses of the lemma. Then it follows that there exist $\sigma$-stages $t_{1}$ and $t_{2}$ with $s_{0}<t_{1}+1 \leq t_{2} \leq t$ such $m x(\sigma, i)$ was appointed at stage $t_{1}+1$ and $s(\sigma, i)$ was appointed at $t_{2}+1$. Now at stage $t_{1}+1$ we set $b\left(\sigma, t_{1}+1\right)$ to be large and fresh and 
in particular $b\left(\sigma, t_{1}+1\right)>m x(\sigma, i) .\left(b\left(\sigma, t_{1}+1\right)\right.$ is appointed in Step 5 after $x(\sigma, i)$ has been appointed and completely defined.) By definition of $\sigma$-stage $t_{2}$ will be the least $\sigma$-stage with $1(e, s)>b\left(\sigma, t_{1}+1\right)$. By choice of $s_{0}$ we know that any follower appointed at stages $v$ with $t_{1}+1<v \leq t_{2}$ must have guess $\tau$ where $\sigma \leq_{L} \tau$ but $\sigma \not \subset \tau$ and $\tau \not \subset \sigma$. Also, any follower appointed at stage $t_{1}+1$ is in $x(\sigma, i)$ and so is $\leq m(\sigma, i)$. By cancellation in Step 1, this means that there are no followers $z$ with

(3.16) $m x(\sigma, i)<z \leq P \quad$ where $P=\max \left\{u\left(\Phi_{e, t_{2}}\left(A_{t_{2}} ; y\right)\right): y \leq s(\sigma, i)\right\}$.

Suppose that (3.15) fails. First we claim that there must be some follower $z$ with

(3.17) $m x(\sigma, i)<z \leq N \quad$ where $N=\max \left\{u\left(\Phi_{e, t}\left(A_{t} ; y\right)\right): y \leq s(\sigma, i)\right\}$, and such that $z$ enters $A_{q}-A_{t}$.

After all, $N$ is also a use function so if $A_{q}[N]=A_{t}[N]$ then for all $y \leq s(\sigma, i)$ we see $\Phi_{e, t}\left(A_{t} ; y\right)=\Phi_{e, q}\left(A_{q} ; y\right)=\left(W_{e, t} \oplus V_{e, t}\right)(y)$. However, if we suppose $A_{q}[N] \neq A_{t}[N]$ by construction some follower $\leq N$ must enter $A_{q}-A_{t}$.

We shall now argue that no such $z$ can exist. Since $z$ is uncancelled at stage $t$ and $z \leq N, z$ must have guess $\gamma \supset \sigma$ and must have been appointed at stage $t_{3}+1$ with $t_{3}$ a $\sigma$-stage and by (3.16), $t_{2} \leq t_{3}<t$. We claim that since $z$ still exists at stage $t$, it must be the case that

$$
A_{t_{3}}[m x(\sigma, i)]=A_{t}[m x(\sigma, i)] \text {. }
$$

If (3.18) fails then some follower $<m x(\sigma, i)$ enters $A$ after stage $t_{3}$ but before stage $t$. Since any such follower must have higher priority than $z$, its entry into $A$ would cancel $z$ (as a follower). But then (3.17) would fail since $z \in A_{q}-A_{t}$. Hence (3.18) holds. Now since $t_{3}$ was a $\sigma$-stage, we know

$$
z>M \text { where } M=\max \left\{u\left(\Phi_{e, t_{3}}\left(A_{t_{3}} ; y\right)\right): y \leq s(\sigma, i)\right\} .
$$

Again by the reasoning used to establish (3.16) there are no followers $g$ with $m x(\sigma, i)<g \leq M$. Now by (3.18) this means that

$$
A_{t_{3}}[M]=A_{t}[M] \text {. }
$$

This, in turn, implies that $M=N$ (computations are unchanged) and now we obtain the desired contradiction: (3.20) and (3.17) say that $z \leq N=M$ yet (3.18) says $z>M$. The lemma now follows. 
(3.21) LEMMA. Let $t$ and $q$ be $\sigma$-stages with $q>t>s_{0}$ and $s(\sigma, i)$ defined at stage $t+1$. Then $\left(W_{e, t} \oplus V_{e, t}\right)[s(\sigma, i)]=\left(W_{e, q} \oplus V_{e, q}\right)[s(\sigma, i)]$ implies $A_{t}[m x(\sigma, i)]=A_{q}[m x(\sigma, i)]$.

Proof. Suppose the lemma fails. Let $t, q$ be as above. We know that if $A_{t}[m x(\sigma, i)] \neq A_{q}[m x(\sigma, i)]$ some (least) follower $z \leq m x(\sigma, i)$ enters $A_{q}$ $-A_{t}$. Let $t_{1} \leq t$ be the $\sigma$-stage where $s(\sigma, i)$ was appointed at stage $t_{1}+1$.

Now there exist $\sigma$-stages $t_{2}$ and $t_{3}$ with $s_{0}<t_{2}<t_{3} \leq t_{1}$ such that $z$ was appointed at stage $t_{2}+1$ and $t_{2}=\operatorname{ls}\left(\sigma, t_{3}\right)$. By the same reasoning as (3.16), we know there is no follower $g$ with

$$
\begin{aligned}
z<g \leq P \quad & \text { where } P=\max \left\{u\left(\Phi_{e, t_{3}}\left(A_{t_{3}} ; y\right)\right): y \leq D\right\} \\
& \text { where } D=\max \left\{u\left(\Gamma_{e, t_{3}}\left(W_{e, t_{3}} \oplus V_{e, t_{3}} ; q\right)\right): q \leq z\right\}
\end{aligned}
$$

(3.22) follows because we reset $b\left(\sigma, t_{2}+1\right)$ to exceed $z$. Now since $z$ is uncancelled at stage $t$, we know that no follower less than $z$ can have entered $A$ between stages $t_{2}$ and $t$, since the entry of such followers would automatically cancel $z$. It follows that

$$
A_{t}[P]=A_{t_{3}}[P] \text {. }
$$

But now (3.23) and the definition of $P$ in (3.22) mean that

$$
\left(W_{e, t} \oplus V_{e, t}\right)[D]=\left(W_{e, t_{3}} \oplus V_{e, t_{3}}\right)[D] \text {. }
$$

Now as $D \leq s(\sigma, i)$ since $t_{3} \leq t_{1}$, we see that the hypotheses of the lemma mean

$$
\left(W_{e, t_{3}} \oplus V_{e, t_{3}}\right)[D]=\left(W_{e, q} \oplus V_{e, q}\right)[D] .
$$

This means that the $\Gamma_{e}$-computations involved in $D$ in (3.22) are unchanged at stage $q$, and in particular

$$
\Gamma_{e, t_{3}}\left(W_{e, t_{3}} \oplus V_{e, t_{3}} ; z\right)=\Gamma_{e, q}\left(W_{e, q} \oplus V_{e, q} ; z\right)=0 .
$$

Therefore $z \notin A_{q}$. This specifically contradicts the assumption that $z \in A_{q}-A_{t}$, and so establishes the lemma.

If we put (3.21) and (3.15) together, summarizing, we see

(3.27) LemMa. Let $t$ and $q$ be $\sigma$-stages with $s_{0}<t<q$ and $s(\sigma, i)$ defined at stage $t$. Then

(i) $\left(W_{e, q} \oplus V_{e, q}\right)[s(\sigma, i)] \neq\left(W_{e, t} \oplus V_{e, t}\right)[s(\sigma, i)]$ iff $A_{q}[m x(\sigma, i)] \neq$ $A_{t}[\operatorname{mx}(\sigma, i)]$. 
(ii) If $A_{q} \neq A_{t}$, the least numbers to enter $A_{q}-A_{t}$ are $y$ and $y+1$ where $y$ is a follower and for some least $j \leq i, y+1=m x(\sigma, j)$.

(iii) Suppose $A_{q} \neq A_{t}$ and $x(\sigma, i)$ is defined at stage $t$. Suppose further that for $j$ given by (ii) we have $j<i$. Then there exist at least two numbers $g_{i}, h_{i} \neq y, y+1$ with $g_{i}, h_{i} \in x(\sigma, i)$ and $g_{i}, h_{i} \in A_{q}-A_{t}$.

(iv) The construction does not break down at Step 6 and $Q \sqcup R={ }^{*} A$.

Proof. (i) simply combines (3.21) and (3.15). (ii) is an easy induction. (iii) is exactly what the mechanics of the construction were set up to ensure, and follows immediately by what happens in Step 4, Case 2 of the construction. Finally (i) implies (iv).

(3.28) LEMMA. $W_{e} \equiv_{T} Q_{\sigma}$ and $V_{e} \equiv{ }_{T} R_{\sigma}$.

Proof. We verify that $W_{e} \equiv{ }_{T} Q_{\sigma}$. The fact that $V_{e} \equiv{ }_{T} R_{\sigma}$ is proved mutatis mutandis. First $Q_{e} \leq{ }_{T} W_{e}$. To determine $Q_{e}[z]$ for $z \in \omega$, find the least $\sigma$-stage $t>s_{0}$ with $s(\sigma, z)$ defined at stage $t$ and $W_{e, t}[s(\sigma, z)]=$ $W_{e}[s(\sigma, z)]$. Direct analysis of Step 6 and (3.27)(i) imply that $Q_{\sigma, t+1}[m x(\sigma, z)]=Q_{\sigma}[m x(\sigma, z)]$ and so $Q_{\sigma, t+1}[z]=Q_{\sigma}[z]$ since $z \leq$ $m x(\sigma, z)$.

The reverse direction is more difficult. We claim similarly that if $t$ is the least $\sigma$-stage with $t>s_{0}$ and $Q_{\sigma, t+1}[m x(\sigma, z)]=Q_{\sigma}[m x(\sigma, z)]$ then $W_{e, t}[s(\sigma, z)]=W_{e}[s(\sigma, z)]$.

Suppose not. Let $q>t$ be the least $\sigma$-stage with $W_{e, t}[s(\sigma, z)] \neq$ $W_{e, q}[s(\sigma, z)]$, and let $t_{1}=1 \mathrm{~s}(\sigma, q)$. By (3.27)(i) we see that $A_{q}[m x(\sigma, z)]$ $\neq A_{t_{1}}[m x(\sigma, z)]$. There exists some least $g \leq z$ such that

$$
\left\{\begin{array}{l}
A_{t_{1}}[m x(\sigma, g)] \neq A_{q}[m x(\sigma, g)] \text { and } \\
W_{e, t}[s(\sigma, g)] \neq W_{e}[s(\sigma, g)] .
\end{array}\right.
$$

By construction, Step 6, and (3.27)(iii) we know that for at least one $y \in x(\sigma, g)$, we have $y \in Q_{\sigma, q+1}-Q_{\sigma, q}$. But then $Q_{\sigma, t+1}[m x(\sigma, z)] \neq$ $Q_{\sigma}[m x(\sigma, z)]$ after all. (This is the whole point of the $x(\sigma, g)$ in the construction.)

REMARK. We remark that $A$ constructed as above has contiguous r.e. degree (i.e. consists of a single r.e. wtt-degree). This follows by (3.27)(i). Not every r.e. set of contiguous degree is USP. Take for example a hypersimple r.e. set of continguous degree. However, every r.e. contiguous degree does contain an SUSP r.e. set. This is proved in [AF] where 
Ambos-Spies and Fejer actually show that an r.e. cylinder of contiguous degree is SUSP. We do not know if every contiguous degree contains a simple SUSP r.e. set.

(3.30) Corollary. (S)USP is not invariant under automorphisms of the lattice of r.e. sets.

Proof. It is well known that there are promptly simple hypersimple r.e. low sets. For example, by [AJSS], the deficiency set of a (low) promptly simple r.e. set if (low and) promptly simple. By Dekker [De], the deficiency set of an r.e. nonrecursive set is hypersimple.

Now let $B$ be a hypersimple low promply simple r.e. set. By Theorem (3.2) $B$ is non-USP. Let $A$ be an SUSP promptly simple low r.e. set given by (3.11). By Maass [Ma] there is an automorphism of the lattice of r.e. sets taking $A$ to $B$.

We remark that Martin's methods (cf. [So1]) of constructing automorphisms appear sufficient to prove the above result directly if we do not wish to use Maass's very powerful result [Ma] and hence to use the full automorphism machinery.

We finally remark that in [Do2], [DS1] and [DW], stronger "nonsplitting" properties of r.e. sets were examined. For example in [DW], Downey and Welch construct r.e. sets $A$ and $B$ with $\varnothing<{ }_{T} B<{ }_{T} A$ such that if $A_{1} \sqcup A_{2}=A$ is an r.e. splitting of $A$, then $A_{1} \leq{ }_{T} B$ implies $A_{1} \equiv_{T} \varnothing . A$ is said to have the antisplitting property. It is unclear if (for example) maximal sets have the antisplitting property and this would seem an interesting line of investigation to pursue.

\section{REFERENCES}

[B] K. Ambos-Spies, Contiguous r.e. degrees, Logic Colloquium '83, (ed. M. Richter) Springer-Verlag lecture notes, 1104 Springer-Verlag, New York (1984), 1-37.

[AS2] _ Anti-mitotic recursively enumerable sets, Z. Math. Logik Grund. Math., 31 (1985), 461-477.

[AF] K. Ambos-Spies and P. Fejer, Degree theoretic splitting properties of r.e. sets, (to appear).

[AJSS] K. Ambos-Spies, C. Jockusch, R. Soare and R. Shore, An algebraic decomposition of the recursively enumerable degrees and the coincidence of several degree classes with the promptly simple degrees, Trans. Amer. Math. Soc., 281 (1984), 109-128.

[De] J. C. E. Dekker, A theorem on hypersimple sets, Proc. Amer. Math. Soc., 5 (1954), 791-796.

[Do1] R. G. Downey, The degrees of r.e. sets without the universal splitting property, Trans. Amer. Math. Soc., 291 (1985), 337-351. 
[Do2] _ Localization of a theorem of Ambos-Spies and the strong antisplitting property, Illinois J. Math., (to appear).

[DS1] R. G. Downey and M. Stob, Structural interactions of the recursively enumerable Tand $W$-degrees, Annals Pure and Applied Logic, 31 (1986), 205-236.

[DS2] __ Automorphisms of the lattice of recursively enumerable sets: Orbits, in preparation.

[DW] R. G. Downey and L. V. Welch, Splitting properties of r.e. sets and degrees, J. Symbolic Logic, 51 (1986), 88-109.

[La1] A. Lachlan, Lower bounds for pairs of r.e. degrees, Proc. London Math. Soc., 16 (1966), 309-316.

[La2] The priority method I, Z. Math. Logik Grund. Math., 13 (1967), 1-10.

[La3] A recursively enumerable degree which will not split over all lesser ones, Ann. Math. Logic, 9 (1975), 307-365.

[Ld1] R. Ladner, Mitotic recursively enumerable sets, J. Symbolic Logic, 38 (1973), 199-211.

[Ld2] _ A completely mitotic nonrecursive r.e. degree, Trans. Amer. Math. Soc., 184 (1973), 479-507.

[LR1] M. Lerman and J. B. Remmel, The universal splitting property, in Logic Colloquium '80 (ed. Van Dalen, Lascar and Smiley) Studies in Logic 108, North-Holland, Amsterdam (1982), 181-209.

[LR2] _ The universal splitting property, II, J. Symbolic Logic, 49 (1984), 137-150.

[Ma] W. Maass, Recursively enumerable generic sets, J. Symbolic Logic, 47 (1982), 809-823.

[Po] E. Post, Recursively enumerable sets of integers and their decision problems, Bull. Amer. Math. Soc., 50 (1944), 284-316.

[So1] R. I. Soare, Automorphisms of the lattice of recursively enumerable sets, part I: maximal sets, Ann. Math., 100 (1974), 80-120.

[So2] _ The infinite injury priority method, J. Symbolic Logic, 41 (1976), 513-530.

[So3] _ Tree arguments in recursion theory and the $\mathbf{O}^{\prime \prime \prime}$-priority method, in Recursion Theory (ed. A. Nerode and R. Shore) Amer. Math. Soc. Publication, Providence, R. I. (1985), 53-106.

[So4] __ Recursively Enumerable Sets and Degrees, (to appear) Springer-Verlag Omega Series.

[St] M. Stob, Wtt-degrees and T-degrees of r.e. sets, J. Symbolic Logic, 48 (1983), 921-930.

Received October 10, 1985 and in revised form April 11, 1986. This research was carried out whilst the author held a position in the department of Mathematics at the University of Illinois, Urbana, U.S.A.

\section{VICTORIA UNIVERSITY}

Private Bag

Wellington, NeW Zealand 



\section{PACIFIC JOURNAL OF MATHEMATICS EDITORS}

\author{
V. S. VARADARAJAN \\ (Managing Editor) \\ University of California \\ Los Angeles, CA 90024 \\ HeRbert Clemens \\ University of Utah \\ Salt Lake City, UT 84112 \\ R. FINN \\ Stanford University \\ Stanford, CA 94305
}

\author{
HERMANN FLASCHKA \\ University of Arizona \\ Tucson, AZ 85721 \\ RAMESH A. GANGOLLI \\ University of Washington \\ Seattle, WA 98195 \\ VAUGHAN F. R. JONES \\ University of California \\ Berkeley, CA 94720 \\ ROBION KIRBY \\ University of California \\ Berkeley, CA 94720
}

\author{
C. C. MOORE \\ University of California \\ Berkeley, CA 94720 \\ H. SAMELSON \\ Stanford University \\ Stanford, CA 94305 \\ HAROLD STARK \\ University of California, San Diego \\ La Jolla, CA 92093
}

\section{ASSOCIATE EDITORS}
R. Arens
E. F. BECKENBACH
B. H. NEUMANN
F. WOLF
K. YosHIDA (1906-1982)

\section{SUPPORTING INSTITUTIONS}

UNIVERSITY OF ARIZONA
UNIVERSITY OF BRITISH COLUMBIA
CALIFORNIA INSTITUTE OF TECHN
UNIVERSITY OF CALIFORNIA
MONTANA STATE UNIVERSITY
UNIVERSITY OF NEVADA, RENO
NEW MEXICO STATE UNIVERSITY
OREGON STATE UNIVERSITY

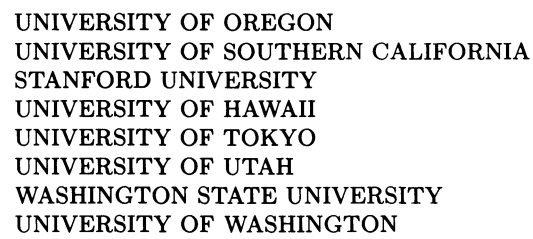

The Supporting Institutions listed above contribute to the cost of publication of this Journal, but they are not owners or publishers and have no responsibility for its content or policies.

Mathematical papers intended for publication in the Pacific Journal of Mathematics should be in typed form or offset-reproduced (not dittoed), double spaced with large margins. Please do not use built up fractions in the text of the manuscript. However, you may use them in the displayed equations. Underline Greek letters in red, German in green, and script in blue. The first paragraph must be capable of being used separately as a synopsis of the entire paper. In particular it should contain no bibliographic references. Please propose a heading for the odd numbered pages of less than 35 characters. Manuscripts, in triplicate, may be sent to any one of the editors. Please classify according to the scheme of Math. Reviews, Index to Vol. 39. Supply name and address of author to whom proofs should be sent. All other communications should be addressed to the managing editor, or Elaine Barth, University of California, Los Angeles, California 90024.

There are page-charges associated with articles appearing in the Pacific Journal of Mathematics. These charges are expected to be paid by the author's University, Government Agency or Company. If the author or authors do not have access to such Institutional support these charges are waived. Single authors will receive 50 free reprints; joint authors will receive a total of 100 free reprints. Additional copies may be obtained at cost in multiples of 50 .

The Pacific Journal of Mathematics is issued monthly as of January 1966. Regular subscription rate: $\$ 190.00$ a year (5 Vols., 10 issues). Special rate: $\$ 95.00$ a year to individual members of supporting institutions.

Subscriptions, orders for numbers issued in the last three calendar years, and changes of address should be sent to Pacific Journal of Mathematics, P.O. Box 969, Carmel Valley, CA 93924, U.S.A. Old back numbers obtainable from Kraus Periodicals Co., Route 100, Millwood, NY 10546.

The Pacific Journal of Mathematics at P.O. Box 969, Carmel Valley, CA 93924 (ISSN 0030-8730) publishes 5 volumes per year. Application to mail at Second-class postage rates is pending at Carmel Valley, California, and additional mailing offices. Postmaster: send address changes to Pacific Journal of Mathematics, P.O. Box 969, Carmel Valley, CA 93924.

PUBLISHED BY PACIFIC JOURNAL OF MATHEMATICS, A NON-PROFIT CORPORATION Copyright (C) 1987 by Pacific Journal of Mathematics 


\section{Pacific Journal of Mathematics \\ Vol. 127, No. $2 \quad$ February, 1987}

Richard Martin Aron and Robert Henry Lohman, A geometric function determined by extreme points of the unit ball of a normed space .... . . 209

Kari Astala and M. S. Ramanujan, $(s)$-nuclear sets and operators $\ldots \ldots \ldots 233$ Earl Robert Berkson, Thomas Alastair Gillespie and Paul Scott Muhly, Analyticity and spectral decompositions of $L^{p}$ for compact abelian

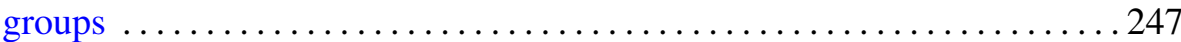

Ronald P. Brown, Real closures of fields at orderings of higher level . . . . . 261

Ronald P. Brown, The behavior of chains of orderings under field

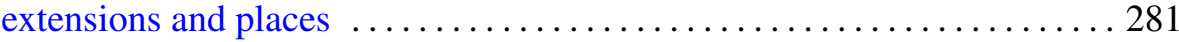

Rodney Graham Downey, Subsets of hypersimple sets .............. 299

Manfred Droste and Saharon Shelah, On the universality of systems of

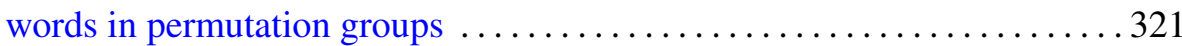

Hidenori Fujiwara, Représentations monomiales des groupes de Lie

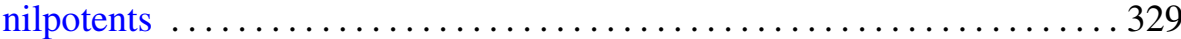

Rupert Lasser, Convolution semigroups on hypergroups $\ldots \ldots \ldots \ldots \ldots 35$

Marcus Marlene Marsh, $u$-mappings on trees $\ldots \ldots \ldots \ldots \ldots \ldots \ldots \ldots \ldots \ldots$

Peter Andrew Symonds, Localization in the classification of flat

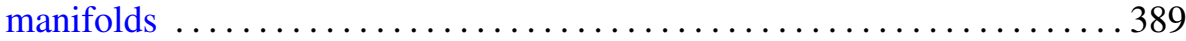

